\title{
Numerical solution of second order ODE directly by two point block backward differentiation formula
}

\begin{abstract}
Direct Two Point Block Backward Differentiation Formula, (BBDF2) for solving second order ordinary differential equations (ODEs) will be presented throughout this paper. The method is derived by differentiating the interpolating polynomial using three back values. In BBDF2, two approximate solutions are produced simultaneously at each step of integration. The method derived is implemented by using fixed step size and the numerical results that follow demonstrate the advantage of the direct method as compared to the reduction method.
\end{abstract}

Keyword: Two point block backward differentiation formula; Second order ODE 